\title{
CHANGES IN THE FEMORAL HEAD UNDERLYING A VITALLIUM GUP
}

\author{
Alexander Gibson and Thomas H. Williams, Winnipeg, Canada \\ From the Departments of Orthopaedic Surgery and Pathology, Deer Lodge Hospital
}

The opportunity of examining the head of a femur that has been subjected to vitallium cup arthroplasty occurs but seldom. We have observed two cases, each with a very different post-operative history.

Case 1-Housewife, aged sixty-three years. Vitallium cup arthroplasty was performed in March 1941 for osteoarthritis of the right hip. One year previously the right breast had been removed for carcinoma. The operation was followed by thrombophlebitis in the right leg.

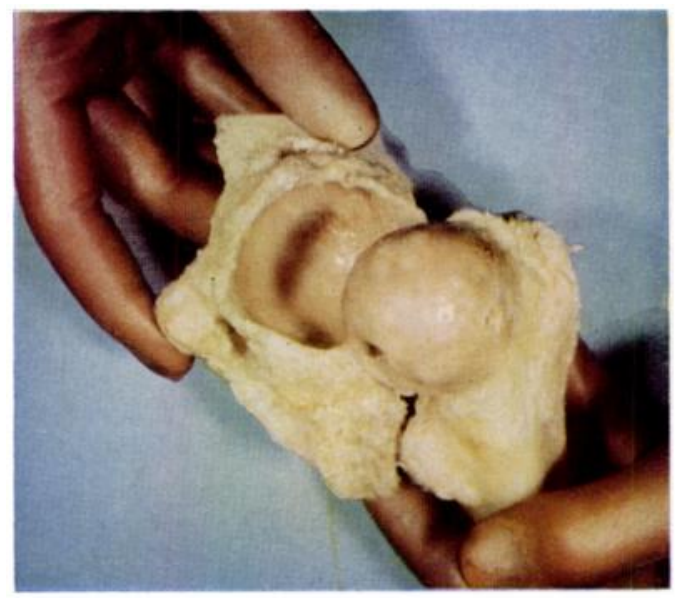

FIG. 1

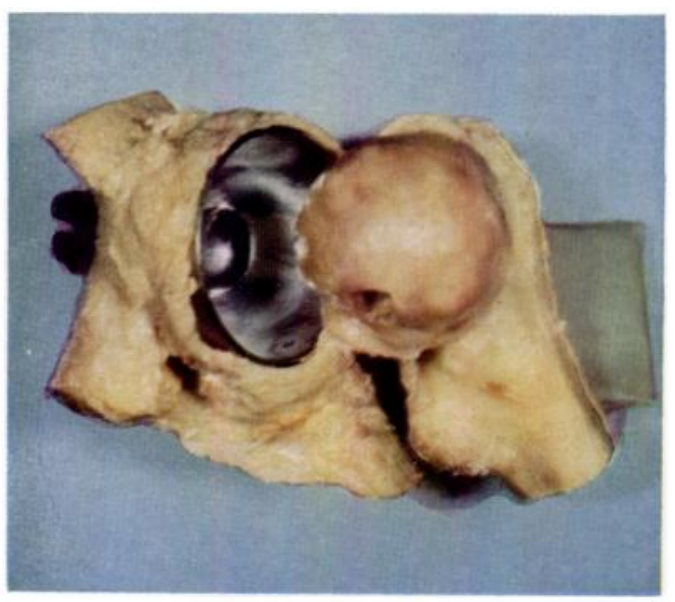

FIG. 2

Case 1-Macroscopic appearance of the joint surfaces one year after cup arthroplasty.

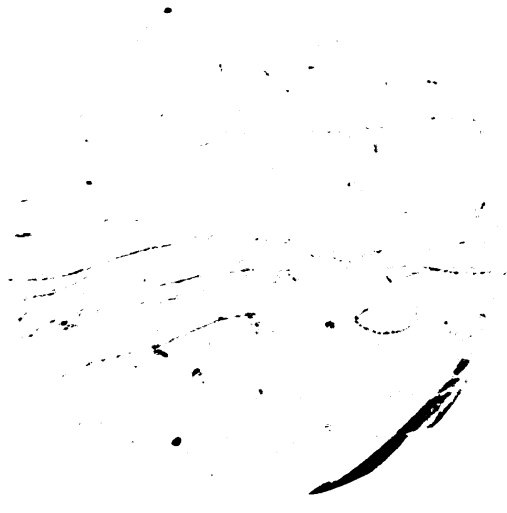

FIG. 3

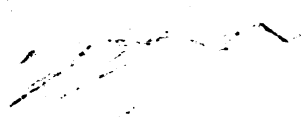

FIG. 4

Case 1-Microscopic sections of the femoral head. Figure 3 shows cancellous bone with osteoblasts; no cartilage cells. Figure 4 shows parallel lavers of collagenous fibrous tissue at the surface, but no cartilage cells.

When she was readmitted to hospital in 194: there was evidence of carcinomatous metastases in the supraclavicular glands, within the thorax, and in the fourth and sixth thoracic vertebral 


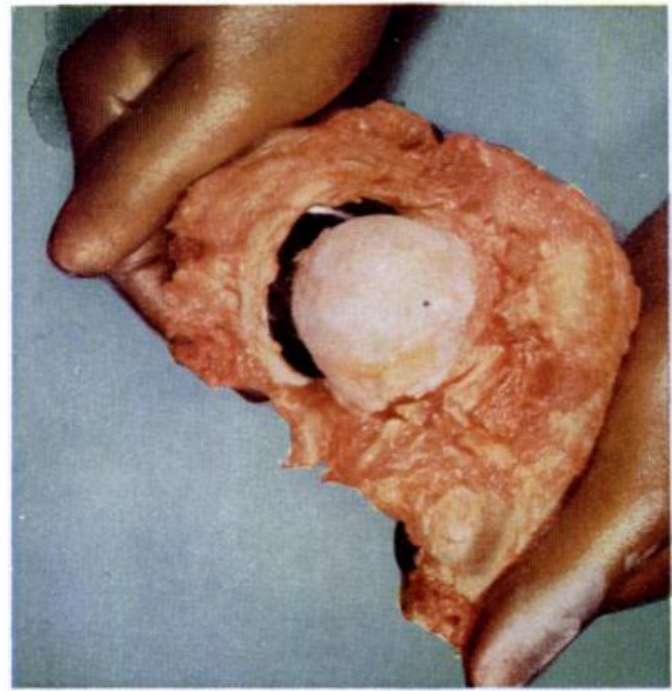

Fig. 5

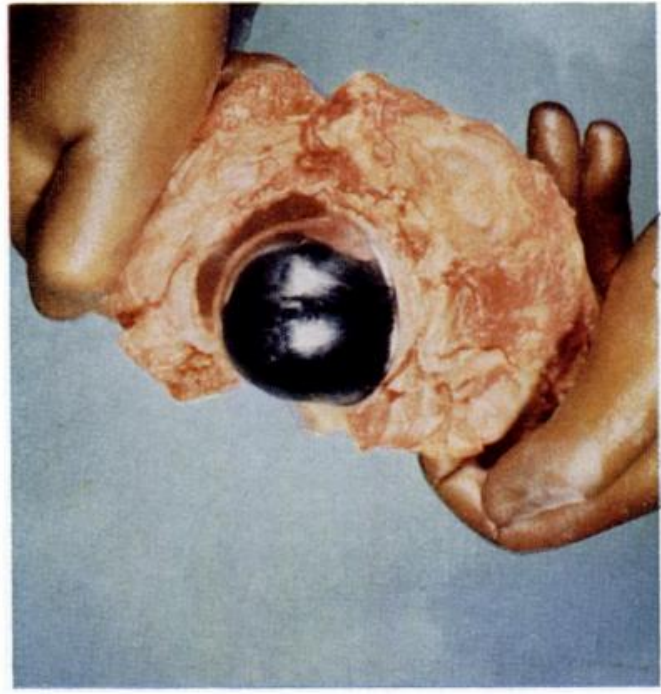

FIG. 6

Case 2-.Macroscopic appearance of the joint surfaces three years and eight months after cup arthroplasty.

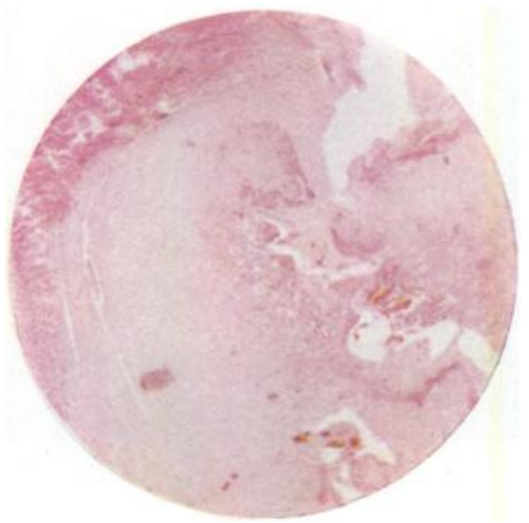

Fig. 7

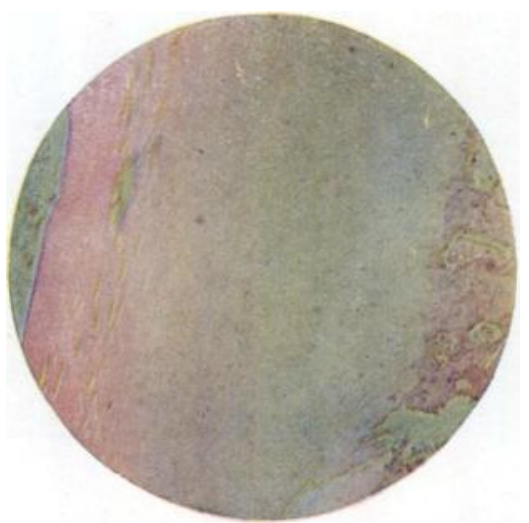

FIG. 8

Case 2-Microscopic sections of the femoral head. Figure 7 shows cancellous bone and cartilage cells. Figure 8 shows the articular surface in which cartilage cells are present.

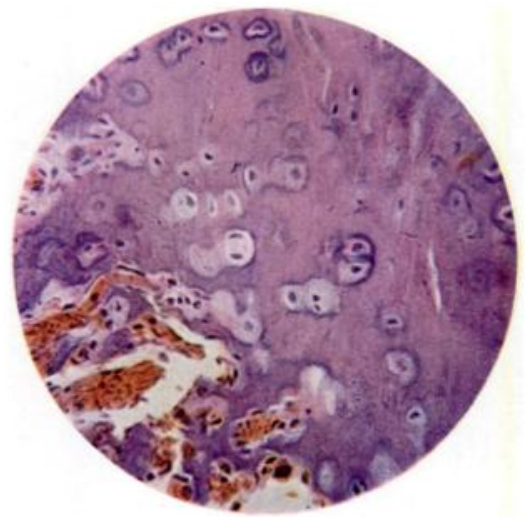

FIG. 9

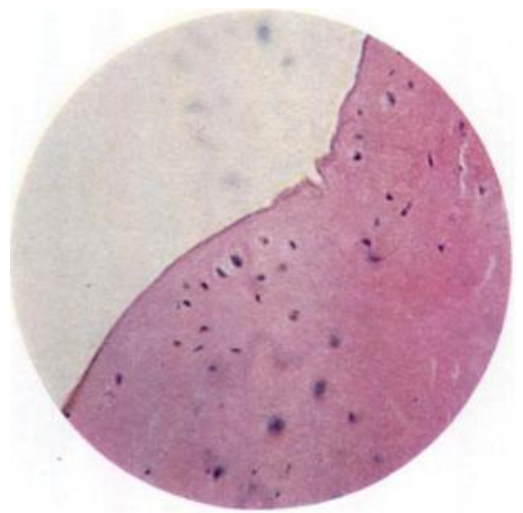

Fig. 10

Case 2. Figure 9-High-power magnification of the area shown at the centre in Figure 7. Note cartilage cells laid down de novo over bone made raw at time of operation. Figure 10-Section from acetabular rim showing cartilage cells at articular surface. 
bodies. She died soon after admission, one year after the cup arthroplasty had been performed.

Autopsy-Death was from generalised carcinomatosis. The joint capsule of the right hip was continuous and normal in thickness. The vitallium cup was found in the acetabulum and was movable both in the socket and on the femoral head. There were many exostoses above the acetabulum. The surface of the femoral head was dull pink with irregular pale mottling (Figs. 1 and 2).

Histological examination of the head of the femur (Figs. 3 and 4) showed cancellous bone with marrow cells in the interspaces. One small area of metastatic carcinoma was seen in the marrow. In places there was evidence of deposition of bone by osteoblasts. No cartilage was seen, but the bone was covered directly by collagenous fibrous tissue which was loose at the bone margin and arranged in dense parallel layers below the articular surface. In some places at the surface there was a very loose collagenous degenerative appearance which explained the mottling seen macroscopically (Fig. 4). No cartilage cells were seen at the surface.

Case 2-Musician, aged fifty-eight years. Cup arthroplasty was done in 1946 for osteoarthritis of the right hip joint. The operation was followed by prolonged physiotherapy, and the patient gained an excellent range of movement.* Three years later a cough began; investigations showed bronchogenic carcinoma with supraclavicular metastases. He died in December 1949, three years and eight months after the cup arthroplasty.

Autopsy confirmed that death was due to squamous bronchogenic carcinoma of the right upper lobe with widespread metastases. The joint capsule of the right hip was complete and normal in thickness. The vitallium cup moved freely in the acetabulum, and the femoral head moved freely in the cup. The rounded head of the femur was covered by a smooth bluish surface resembling articular cartilage (Figs. 5 and 6).

Histological examination of sections taken vertically through the head of the femur under the vitallium cup showed cancellous bone with marrow interspaces. The bone terminated in a fairly regular line of ossification merging into cartilage which consisted of an eosinophilic matrix with groups of definite cartilage cells (Figs. 7 to 9). This merged imperceptibly into an articular surface layer where the cells were elongated and parallel to the articular surface. A few cells of stouter type were found in the surface layer. It seemed that there had been replacement of the cartilage after trimming of the head of the femur at operation.

Sections from the rim of the acetabulum (Fig. 10) showed synovial surface tissue at the border merging into articular cartilage with a dense eosinophilic fibrous and muscle base. The cartilage cells along the surface were typical of articular cartilage with paired nuclei.

\section{COMMENT}

In both cases, the articular cartilage covering the head of the femur was removed entirely. In the first case, the patient was an obese elderly woman in poor physical condition. Not much use was made of the arthroplasty; the chief benefit to the patient had been relief from pain. In conformity with this small amount of activity we found no cartilage formed over the head of the femur but only fibrous tissue, which was, in places, unhealthy. In marked contrast, the newly formed joint had been used by the second patient freely and constantly for more than three and a half years. In his case the head of the femur was covered by true articular cartilage, with healthy bone underneath and a smooth articular surface.

The two specimens illustrated seem to demonstrate that tissue form and cellular type correspond to functional demand, and that for the reproduction of articular cartilage, compression plus gliding movement are a necessary combined stimulus.

* A motion picture of this patient was shown at the combined meeting of the American, British and Canadian Orthopaedic Associations in Quebec in 1948.

vol. 33 B, No. 1, FEBRUARY 1951 